\title{
A New Proposal on Reductant Utilization in Flue Gas SCR De- $\mathrm{NO}_{\mathrm{x}}$ Process
}

\author{
Jie Li ${ }^{\text {a }}$, Jian Li \\ Key Laboratory of Beijing on Regional Air Pollution Contro, Beijing 100124, China. \\ alijie8069@126.com
}

\begin{abstract}
A direct injection proposal of ammonia hydroxide employed in Flue Gas SCR De-NOx process was presented and a simulation was implemented by the advanced CFD software Star$\mathrm{CCM}$ to verify the performance of this proposal. The initial equipment investment cost and operation cost could be reduced greatly by employing this proposal which will lead to one of important development directions in flue gas $\mathrm{De}-\mathrm{NO}_{\mathrm{x}}$.
\end{abstract}

Keywords: Flue Gas De-Nitrification (De-NOx), Reductant, Ammonia Hydroxide.

\section{Introduction}

Currently, the control and management on $\mathrm{NO}_{\mathrm{x}}$ has been under the spotlight in the field of air pollution control. Flue gas De- $\mathrm{NO}_{\mathrm{x}}$ has been popular to reduce $\mathrm{NO}_{\mathrm{x}}$ emission in developed countries, and it consists of Selective Catalytic Reduction (SCR), Selective Non-Catalytic Reduction (SNCR) and the combined with SCR and SNCR. These technologies have been employed extensively in flue gas De-NOx projects involved in boiler, kiln and industrial facilities with abundant $\mathrm{NO}_{\mathrm{x}}$ emission. The reductant plays a vital role in flue gas De- $\mathrm{NO}_{x}$ process and its cost impacts directly the overall economic index of De- $\mathrm{NO}_{\mathrm{x}}$ system due to its proportionally largest consumption [1].

Anhydrous ammonia, ammonia hydroxide and urea are the primary sources of the reductant ammonia in De-NO $\mathrm{NO}_{\mathrm{x}}$ system. These sources are with different characteristics. Anhydrous ammonia is generally considered advantageous of economic aspects such as investment, while is inherently in high risk in safety aspects and it is listed in major hazard sources as a dangerous chemical. Hence, there are lots of safety limits on the storage, preparation and delivery of anhydrous ammonia, which restricts the application of anhydrous ammonia greatly in cities [2]. Ammonia hydroxide and urea will normally be recommended once anhydrous ammonia is not on the option list due to safety concerns [3]. Urea is of high security, easy delivery, convenient storage and stable price while with relatively high initial investment cost, operation cost and operation personnel with high skills. Ammonia hydroxide is of low price and only physical change is involved during its gasification. However, its high delivery cost needs to be considered. As a byproduct from chemical industry, ammonia hydroxide is put on top priority while the De- $\mathrm{NO}_{\mathrm{x}}$ projects close to chemical factories select reductant because the delivery cost can be saved greatly. Besides, the post treatment cost on ammonia hydroxide will be saved for the chemical factories. It appears this application is economically beneficiary for both buyer and seller of ammonia hydroxide [4]. With the advantage of high security, low investment and operation cost, and excellent cost performance, the reactant ammonia hydroxide is more suitable for middle and small-scale boiler concerning De- $\mathrm{NO}_{\mathrm{x}}$ [4].

Normally, ammonia hydroxide as a reductant cannot be injected into reactor directly in De- $\mathrm{NO}_{\mathrm{x}}$ system, while is evaporated into ammonia gas by heating devices (steam or heat flue gas) and then injected into De- $\mathrm{NO}_{\mathrm{x}}$ system via ammonia injection grid (AIG). Consequently, special evaporator and AIG need to be prepared, which increases the initial investment cost, heating cost and maintenance cost. Below is the conventional flue gas De- $\mathrm{NO}_{\mathrm{x}}$ process diagram using reductant ammonia hydroxide. 


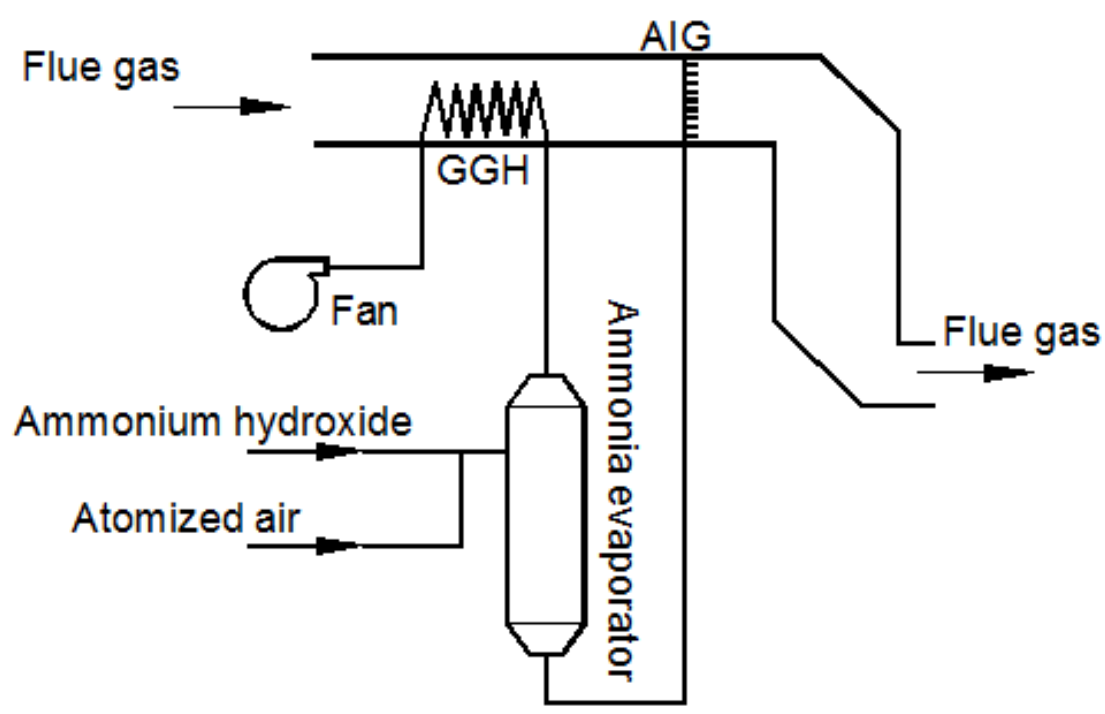

Fig. 1 Conventional Flue Gas De-NOx Process Diagram Using Reductant Ammonia Hydroxide

In view of the high cost of conventional proposal described above, a new proposal without evaporator and AIG is increasingly in urgent need and will be of great application value.

\section{Direct Injection Proposal of Ammonia Hydroxide}

\subsection{Influence Factors and Criterions}

In terms of flues gas De- $\mathrm{NO}_{\mathrm{x}}$ technology, De-NO $\mathrm{N}_{\mathrm{x}}$ efficiency depends on many factors while the concentration distribution of reductant ammonia lists in the most important technology indicators.

To judge the concentration distribution, the maximum Coefficient of Variance (CV) is introduced. If the maximum CV is less than or equal to $5 \%$ at the cross-section $0.5 \mathrm{~m}$ away from the upstream of the first layer of catalyst, a uniform concentration distribution will be deemed to develop.

$\mathrm{CV}$ is defined as

$$
C V=\frac{\sigma_{V}}{\bar{V}} \cdot 100(\%)
$$

In which, $\sigma_{v}=\sqrt{\frac{\sum_{i=1}^{n}\left(V_{i}-\bar{V}\right)^{2}}{n-1}}$

In which, $\bar{V}$ : represents the average concentration value;

VI: the sampling value;

$\mathrm{N}$ : the sampling number.

\subsection{Feasibility Study}

The advanced CFD software Star-CCM+ was employed to verify the feasibility of the direct injection proposal of ammonia hydroxide. Below are critical for this proposal:

a. To ensure complete evaporation of ammonia hydroxide prior to its entering SCR reactor, the ammonia hydroxide injection nozzle should be mounted a certain distance (the evaporation distance of ammonia hydroxide) from the duct of the reactor inlet;

b. The injection nozzles should be arranged on the basis that minimizes the evaporation distance of ammonia hydroxide and makes the ammonia gas distributed as uniform as possible.

In view of the presence of flue gas and ammonia in flue gas De- $\mathrm{NO}_{\mathrm{x}}$ process and the porous catalyst, material transport model, gas-phase turbulence model and porous medium model were employed to fulfill the simulation. The flue gas velocity distribution diagram and ammonia hydroxide residence 
time diagram were presented below by the related simulations involved in velocity distribution, residence time and the ammonia gas concentration distribution at the upstream of catalyst.

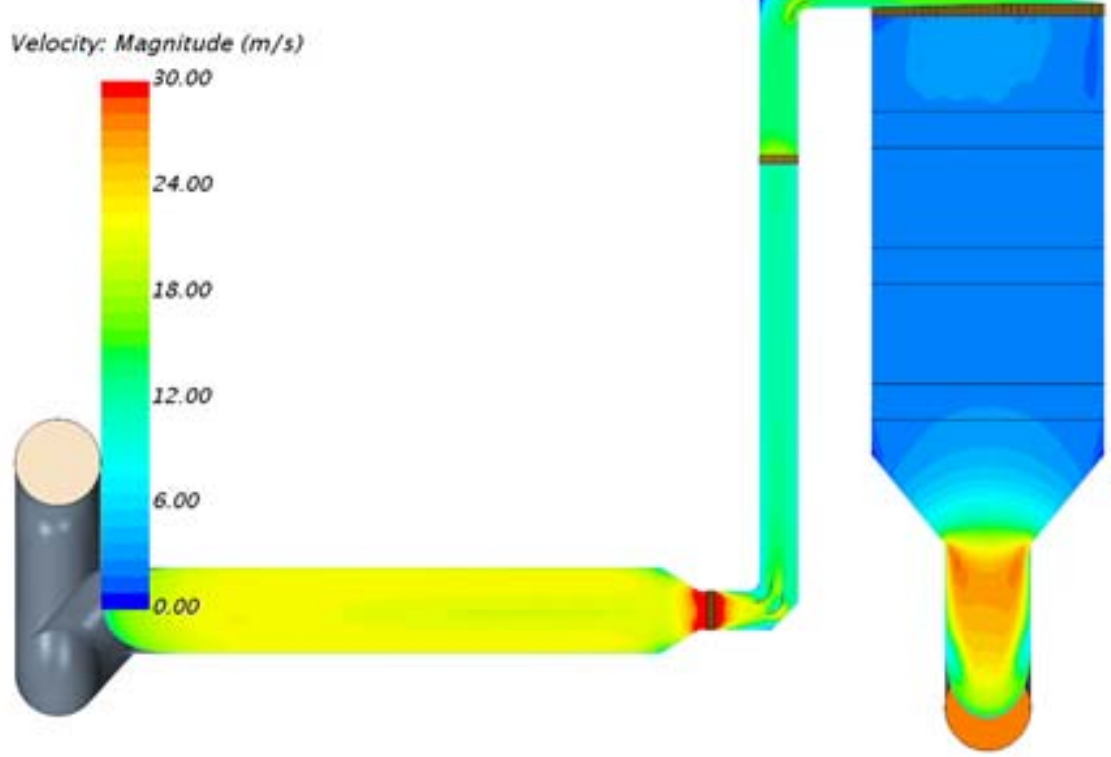

Fig.2 General Velocity Distribution Diagram

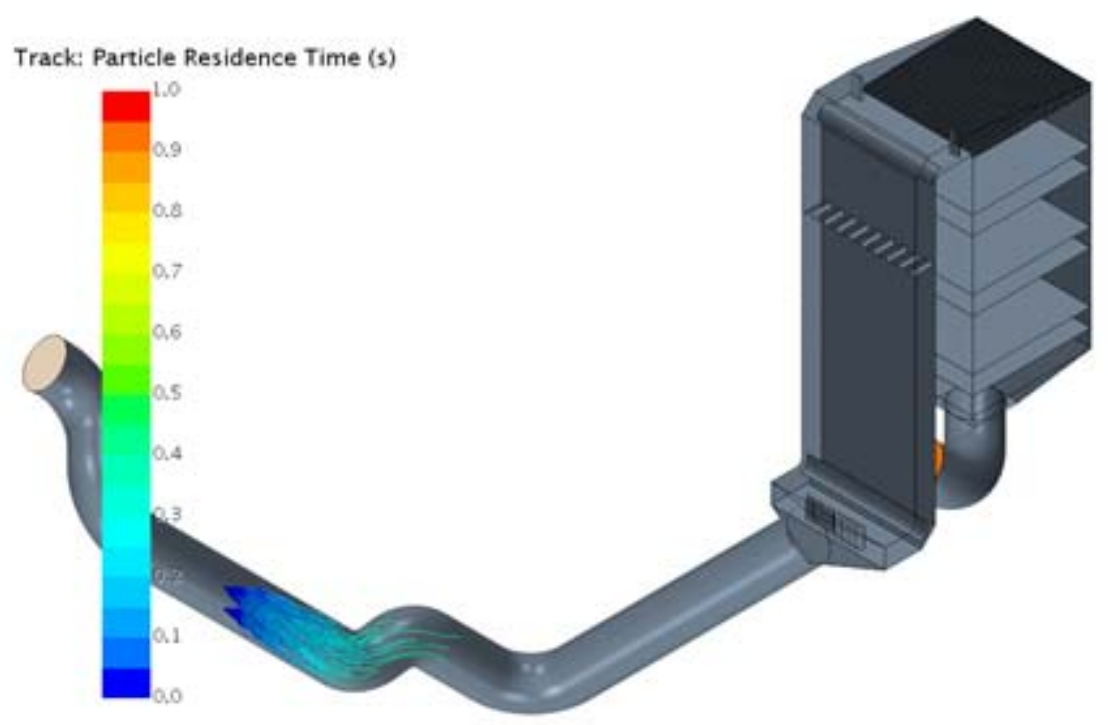

Fig. 3 Ammonia Hydroxide Residence Time Digaram

On the basis of simulation, the arrangement of ammonia hydroxide injection nozzle, injection position and the related parameters of ammonia hydroxide injection devices were adjusted properly to meet the optimal direct injection performance. In terms of Fig.4 Ammonia Gas Concentration Distribution Diagram at the Upstream of Catalyst below, the ammonia hydroxide could be evaporated completely by the heat of flue gas itself without special evaporator and mixed uniformly in the duct; the technical indicator (CV $\leqslant 5 \%$ ) of the concentration field could be met without AIG. 


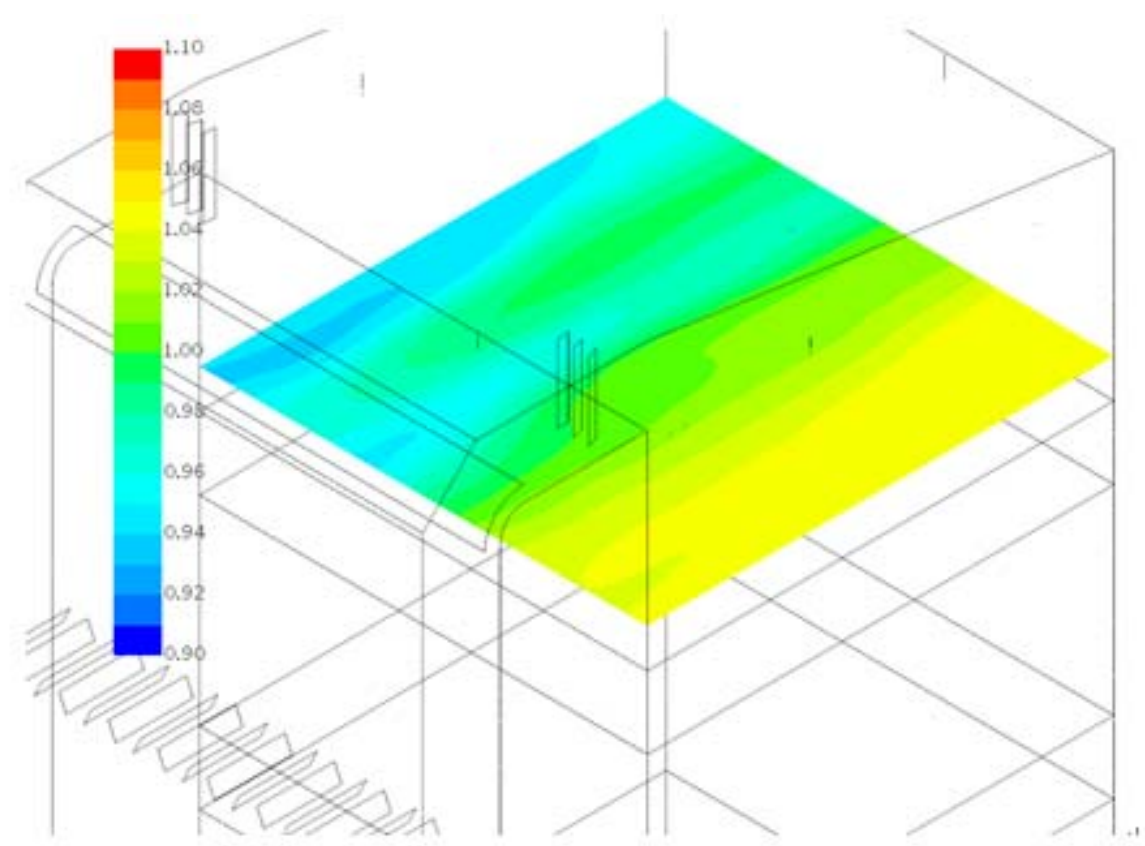

Fig.4 Ammonia Gas Concentration Distribution Diagram at the Upstream of Catalyst

In this simulaiton, the flue gas flow was assumed to be $150,000 \mathrm{Nm} 3 / \mathrm{h}$; the internal diameter of flue gas duct, the diameter and installation angle of injection nozzles were fixed. While ammonia entered the flue gas duct till 28,600mm from the mixing inlet, the ammonia concentration distribution $\mathrm{CV}$ at the upstream of catalyst is $5 \%$; if the mixing distance increased till 41,700 , CV dropped till $3.44 \%$. It appeared this proposal could meet the design requirement of flue gas De- $\mathrm{NO}_{\mathrm{x}}$ projects.

\section{Conclusion}

Compared to the conventional ammonia hydroxide reductant proposal in flue gas De- $\mathrm{NO}_{\mathrm{x}}$ process, the direct injection proposal of ammonia hydroxide without special evaporator and AIG can reduce greatly initial equipment investment cost, heating cost and maintenance cost during phase change from ammonia hydroxide to ammonia gas. Besides, the uniform ammonia concentration distribution can be met. This new proposal will be beneficiary for both economic effectiveness and social effect.

\section{References}

[1]. Wang jiaming. Urea SCR flue gas denitrification technology and its application prospects [J]. Synthesis technology and application, 2013, 01:28-32.

[2]. Liu dehong, gao xiangsheng, li xiaorong. Application of ammonia as reducing agent in SCR denitrification project of small and medium-sized coal-fired boilers [J]. Energy research and utilization, 2015 (02):53-55.

[3]. Wang qi. Selection and economic analysis of reducing agent in flue gas denitrification SCR technology of thermal power plant [J]. Electromechanical information, 2013(18):124-125.

[4]. Wang gang, zhao cuijing. Application of ammonia water in flue gas denitrification system [J]. Boiler manufacturing, 2015 (05):29-31. 